\section{Siameses onfalópagos: presentación de un caso}

\section{RESUMEN}

De todos los embarazos 3\% son embarazos múltiples y los más frecuentes son los dicigóticos del sexo femenino. Sin embargo, un pequeño grupo de ellos son de origen monocigótico con productos unidos en alguna región anatómica. Estos casos atraen la atención de los médicos por su rareza y por las dificultades y dilemas éticos a los que se enfrentan para su tratamiento. Presentamos el caso de unos siameses onfalópagos del sexo masculino a los que se logró separar quirúrgicamente a las siete semanas de vida; uno de ellos sólo sobrevivió seis meses.

Palabras clave: embarazo múltiple, siameses, onfalópagos.
Sheila Yadira Gómez-Murillo ${ }^{1}$ Sara Alejandra Solórzano-Morales² Héctor Macías-Avilés ${ }^{3}$

${ }^{1}$ Residente de tercer año de pediatría.

${ }^{2}$ Médico adscrito al servicio de Imagenología.

${ }^{3}$ Pediatra Neonatólogo. Servicio de neonatología. Instituto Nacional de Pediatría, México, D.F.

\title{
Conjoined omphalopagus twins: a case report
}

\section{ABSTRACT}

Multiple pregnancies are 3\% of the total. The most frequent are dizygotic females, however, a small group of them are of monozygotic conjoined twins in some anatomical area. This attracts the attention of physicians because of the rarity of the condition and the difficulties as well as the ethical dilemmas for their treatment. We report a case of male Siamese omphalopagus. They were separated surgically at seven weeks of age. One of them lived six months.

Key words: Multiple pregnancies, Siamese, Conjoined twins, Omphalopagus.

Recibido: 13 de febrero 2014

Aceptado: 24 de junio 2014

Correspondencia: Dra. Sheila Yadira Gómez Murillo

Instituto Nacional de Pediatría

shgomezm@hotmail.com

Este artículo debe citarse como

Gómez-Murillo SY, Solórzano-Morales SA, MacíasAvilés H. Siameses onfalópagos: presentación de un caso. Acta Pediat Mex 2014;35:469-476. 


\section{INTRODUCCIÓN}

Los embarazos múltiples siempre han sido vistos con gran interés médico debido a la complejidad con la que cursan para llegar a término; implican a aproximadamente $3 \%$ de todos los embarazos, ${ }^{1}$ aunque este porcentaje puede variar de acuerdo con la región geográfica estudiada. Existen dos variedades: gemelos dicigóticos por la fecundación de dos óvulos y monocigóticos por fecundación de un solo óvulo, que comparten la misma información genética. Los más frecuentes son los primeros, del sexo femenino, y ocurren en uno de cada mil embarazos. Esto se atribuye a dos situaciones relevantes: edad materna y el uso de terapias de fertilidad. ${ }^{2}$

Se considera siameses a los individuos monocigóticos y monocoriónicos unidos entre sí en alguna región anatómica, que pueden compartir o no uno o más órganos; son del mismo sexo y genéticamente idénticos, ${ }^{3}$ su incidencia aproximada varía de uno en 50000 a uno en 100000 embarazos múltiples. ${ }^{4}$

Esta situación se puede definir embriológicamente 13 a 14 días después de la concepción. La localización del sitio de unión de los gemelos determinará la nomenclatura que les corresponda. ${ }^{5}$ Las variedades más frecuentes son:

a) Toracópagos: 40 a 75\%; comparten esternón, caja torácica, diafragma, pared abdominal superior y estructuras cardíacas.

b) Onfalópagos: 33\%; presentan fusión esternal y hepática hasta en $80 \%$ de los casos; tienen mayor supervivencia, hasta 53\% después de la separación quirúrgica.

c) Isquiópagos: 6 a 10\%; están involucrados el tronco y la pelvis.

d) Cefalópagos o janicéfalos: 1.7\%; están unidos de cualquier parte del cráneo, a excepción de la cara, comparten hueso, meninges y frecuentemente drenaje venoso. ${ }^{5,6}$

Presentamos un caso de gemelos onfalópagos varones a los que se logró separar a las siete semanas de vida.

\section{CASO CLÍNICO}

Producto de la primera gestación de una madre de 17 años de edad, de un medio rural y sin control prenatal. Los gemelos nacieron por cesárea en un hospital rural después de un intento fallido de parto natural atendido por una partera. Con la cesárea se encontró un embarazo gemelar cuyo peso total fue de $3.9 \mathrm{~kg}$. No se menciona la talla. Apgar del siamés 1, de 3-8; requirió un ciclo de presión positiva intermitente. Siamés 2, Apgar de 7-8. Capurro: 39 semanas de gestación. Se les trasladó a un hospital de tercer nivel a las primeras 12 horas de vida.

Los siameses ingresaron en condiciones estables, sin apoyo ventilatorio, con un catéter umbilical; se mostraban activos, reactivos, tenían fontanela anterior normotensa. Compartían el tórax en la región central. Se auscultaron los ruidos cardiacos de un solo corazón y un soplo sistólico grado II/VI. El abdomen estaba unido en su tercio superior; existía un solo cordón umbilical con dos arterias y una vena, cuatro extremidades superiores y cuatro inferiores (Figuras 1 y 2 ).

\section{SIAMÉS 1}

Se realizó un tamiz metabólico ampliado, ácidos orgánicos en orina. Un ultrasonido toracoabdominal (Figuras 3 y 4) mostró un ángulo de separación de la parrilla costal compartida en la cara anterior. Se observó un hígado unido por un istmo central, sin evidencia de que compartieran estructuras vasculares. Se realizó un estudio de tránsito intestinal (Figura 5). 


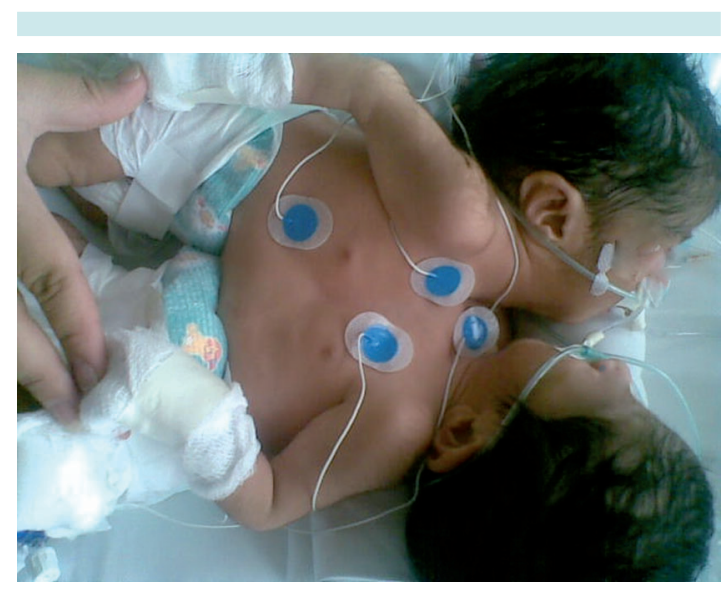

Figura 1. Unión toracoabdominal sin otras alteraciones aparentes.

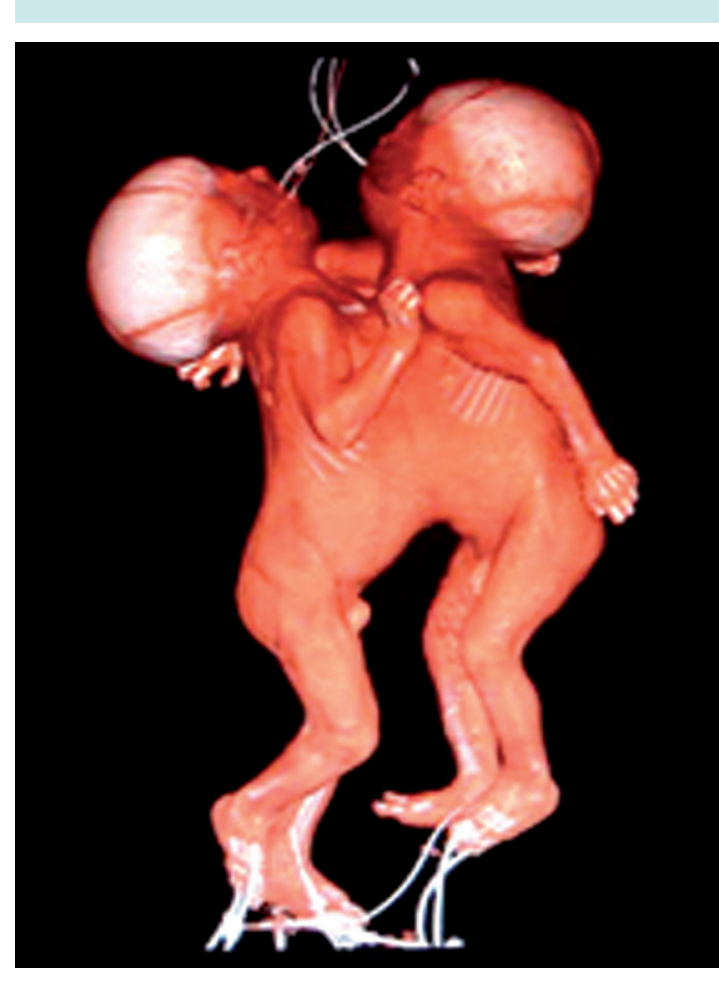

Figura 2. Reconstrucción tridimensional volumétrica de la tomografía computada multicorte, que muestra la superficie ventral de unión de los gemelos desde el esternón hasta el ombligo.
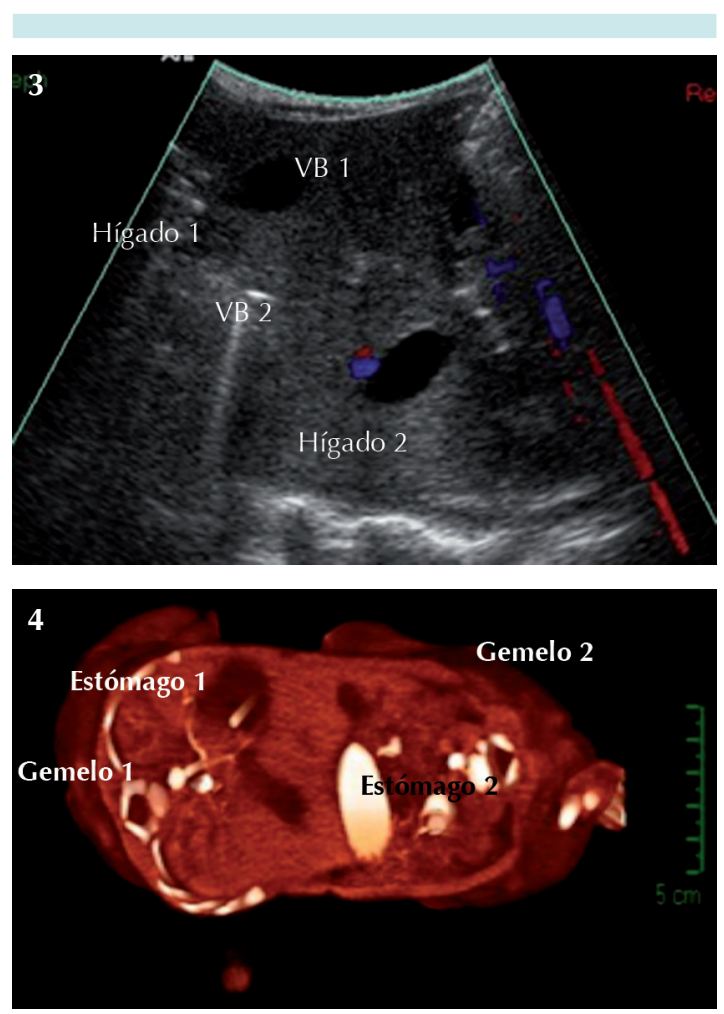

Figura 3 y 4 . Ambos hígados se unían en la línea media, pero no compartían estructuras vasculares o biliares. El ultrasonido demuestra las vesículas biliares independientes.

A los tres días del ingreso se realizó una angiotomografía (Figuras 6 y 7) después de la cual hubo deterioro que requirió fase III de ventilación; hubo hipoxemia persistente con saturación hasta $55 \%$ por oximetría de pulso, a pesar de recibir presión inspiratoria máxima de $29 \mathrm{~cm}$ de $\mathrm{H}_{2} \mathrm{O}$, $\mathrm{FiO}_{2}$ de $100 \%$ y presión positiva al final de la expiración (PEEP) 5. Un ecocardiograma reveló tetralogía de Fallot.

Se hizo una reunión con especialistas de neonatología, neumología, cardiología, cirugía plástica, trasplantes, radiología, comité ético y otros, en la que se concluyó que si se realizara la separación quirúrgica de los gemelos, 


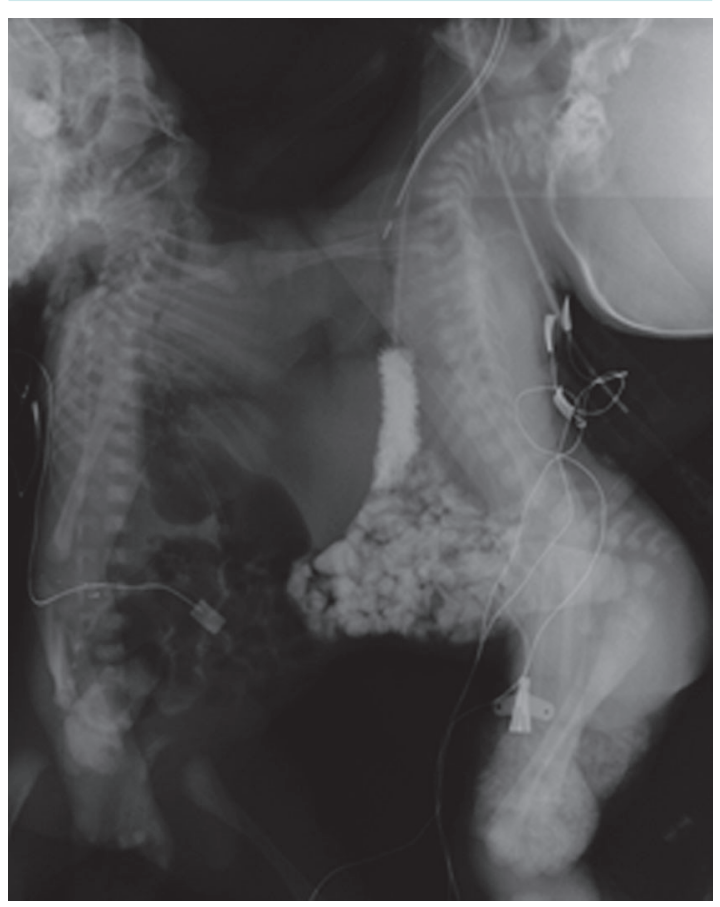

Figura 5. Serie esofagogastroduodenal. Se administró una pequeña cantidad de medio de contraste hidrosoluble a través de la sonda nasogástrica del gemelo 2; no compartían ningún segmento del tubo digestivo como ocurre en $50 \%$ de los casos.

no habría suficiente piel para cubrir las partes expuestas de cada gemelo, por lo cual se decidió que se colocarían expansores tisulares en la cara anterior de ambas parrillas costales. Se realizó el procedimiento quirúrgico posterior al cual presentó inestabilidad hemodinámica. que requirió el uso de aminas; 51 días después del ingreso se llevó a cabo la separación de órganos. La intervención duró diez horas, se colocó una malla de titanio en la pared torácica debido a la ausencia de la mitad de la parrilla costal que compartían los gemelos y no había suficiente piel para una cobertura adecuada.

En el posoperatorio se tuvieron que emplear aminas. Debido a la tetralogía de Fallot y al hecho de haber tenido el tórax abierto, hubo

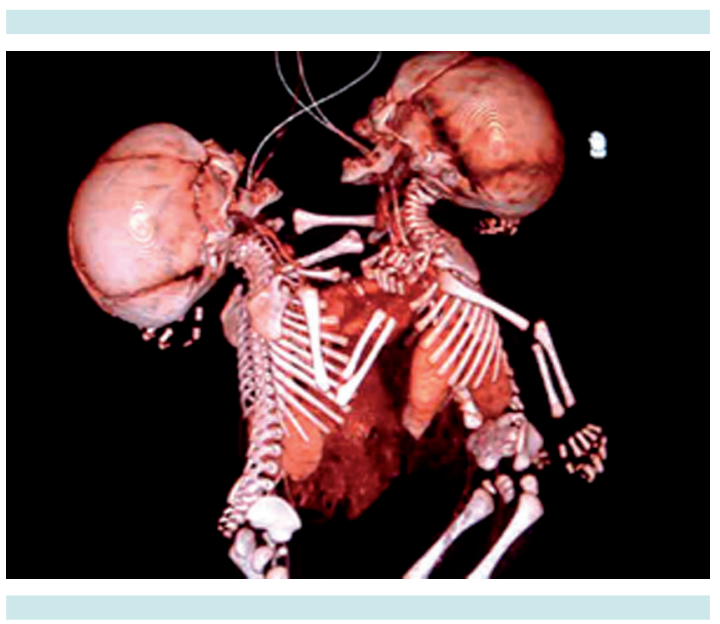

Figura 6. Reconstrucción tridimensional volumétrica que muestra ambos corazones emergiendo centralmente a través de la apertura amplia de las cajas torácicas. Los estómagos separados con sus correspondientes sondas nasogástricas se ubican a la izquierda de cada gemelo (situs solitus).

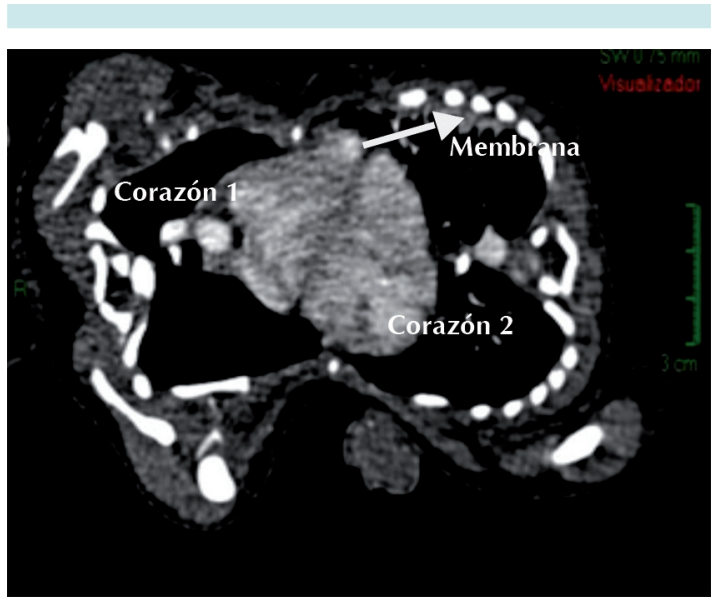

Figura 7. Corte axial de la tomografía computada en fase contrastada del tórax, que muestra la unión de ambos corazones.

compromiso circulatorio y necrosis del tejido circundante a la malla de titanio; se produjo distensión abdominal. La evolución fue tórpida, con anuria 48 horas después de la cirugía, hiperlactamia, hiperpotasemia e hipotensión, a pesar 
del tratamiento médico. Presentó paro cardiaco que no respondió a las maniobras de reanimación.

\section{SIAMÉS 2}

Se le realizaron los mismos estudios que al primer gemelo que presentó reducción del gasto urinario, por lo cual se le dio un diurético. Llamó la atención el incremento del gasto urinario en el siamés 1 , por lo cual se decidió realizar angiotomografía en el siamés 2 para determinar la vasculatura compartida. Después de este estudio, el paciente tuvo paro circulatorio que fue tratado con una dosis de atropina y adrenalina, que dio buen resultado. La evolución fue tórpida, con datos de sepsis e insuficiencia respiratoria que requirió ventilación mecánica durante cinco días.

Se hicieron estudios para establecer un plan quirúrgico: un ecocardiograma del corazón mostró anatomía segmentaria normal, cuya pared ventricular en la porción media reveló movilidad ventricular independiente para ambos siameses. Había discontinuidad del pericardio en la misma zona y un conducto arterial con cortocircuito de izquierda a derecha que no causaba compromiso hemodinámico.

Una vez separados los órganos el paciente fue enviado a la Unidad de Cuidados Intensivos Neonatales, hemodinámicamente inestable; su herida quirúrgica estaba limpia, bien afrontada en la línea media, que iba desde el tórax hasta la parte baja del abdomen. Recibió apoyo con succión continua. Había datos de insuficiencias renal y cardiaca; anuria, taquicardia y pulsos periféricos débiles, con Ilenado capilar lento, lo que requirió solución cristaloide y aminas; eso mejoró los signos vitales, la diuresis y propició la estabilidad hemodinámica.

A las 48 horas de la operación se mantenía en condiciones críticas, con anasarca, febril y con datos de respuesta inflamatoria sistémica.
Un cultivo de tejido circundante a la malla de titanio y de los expansores tisulares fue positivo para Pseudomonas aeruginosa. El Servicio de Infectología indicó tratamiento con piperacilinatazobactam pues el paciente estaba en choque séptico. El Servicio de Inmunología sugirió dar tratamiento con gammaglobulina como terapia coadyuvante.

Once días después de la operación apareció una zona equimótica de $2 \mathrm{~cm}$ de diámetro en el hipocondrio derecho. Un ultrasonido abdominal mostró líquido libre en cavidad y dos colecciones intraabdominales en el flanco derecho y en el hipocondrio izquierdo, respectivamente; sin embargo, las condiciones clínicas del paciente mejoraron, lo que permitió suspender las aminas. Tres días después, se inició la estimulación enteral, con incremento paulatino, hasta alcanzar requerimientos completos por vía enteral y progresando con alimentación hasta iniciar ablactación a los seis meses de edad.

Dos meses después de la intervención quirúrgica hubo dehiscencia de la herida en la zona de colocación de los expansores tisulares de la caja torácica, con exposición de la malla de titanio, de la cual se tomó un cultivo de secreción que mostró crecimiento de Staphylococcus epidermidis y Pseudomonas aeruginosa; sin embargo, el paciente estaba en buenas condiciones generales, tolerando adecuadamente la alimentación por vía oral, con buen crecimiento, afebril, sin datos de respuesta inflamatoria sistémica. Se realizó el aseo de la caja torácica con solución de ciprofloxacina durante tres días. Un nuevo cultivo no mostró agentes infecciosos.

A los 157 días de su ingreso, el siamés dos fue operado nuevamente: recambio de expansores tisulares y rotación de colgajos en caja torácica, después de lo cual tuvo bradicardia e hipotermia; requirió apoyo inotrópico con dobutamina. Debido a la insuficiencia respiratoria se inició 
fase III de ventilación. Apareció una alteración del colgajo con eritema del borde superior y compromiso vascular; 24 horas después hubo sangrado activo en el colgajo e hipoperfusión tisular. El paciente tuvo oliguria a pesar de haber recibido soluciones cristaloides y diurético.

La evolución fue tórpida, con hipoxia, distensión abdominal, insuficiencias respiratoria, cardiovascular, renal, hematológica y gastrohepática. Se le mantuvo con apoyo aminérgico hasta el día 172 de su estancia, cuando tuvo paro cardiaco de un minuto. Se mantuvo hemodinámicamente inestable, con oliguria que llegó hasta la anuria; saturación de $\mathrm{O}_{2}$ entre 80 y $82 \%$, aún cuando había parámetros altos de ventilación, hipotensión, bradicardia, hipoperfusión tisular, piel marmórea, metabólicamente con hiperpotasemia: $\mathrm{K}$ sérico de $7 \mathrm{mEq} / \mathrm{L}$. Requirió gluconato de calcio y soluciones polarizantes. Se le administraron dos dosis de adrenalina sin recuperación. No era posible realizar maniobras de reanimación debido a que la caja torácica estaba cubierta con la malla de titanio. El paciente falleció a las 00.45 horas.

\section{ANÁLISIS}

La frecuencia de los embarazos múltiples ha aumentado considerablemente en los últimos 20 años. Actualmente implica 3\% de los embarazos a nivel mundial. Es necesario identificarlos oportunamente y darles seguimiento; asegurarse de contar con el equipo médico e instrumental idóneos para el momento del nacimiento, pues tienen mayor morbilidad y mortalidad, prematurez, retraso del crecimiento intrauterino y comorbilidades como dismorfias que pueden comprometer la vida de los neonatos. ${ }^{1}$

El 6\% de los gemelos unidos ocurre en gestaciones triples. El sexo femenino es más frecuente que el masculino en proporción de 3:1. El 95\% son gemelos femeninos, lo que puede deberse a retraso de los factores (aún no determinados) de los cigotos XX.7,8

Existen algunas características clínicas que permiten sospechar embarazos de alto riesgo como el tamaño del útero (mayor de lo esperado para la edad gestacional), historia de reproducción asistida y alfafetoproteínas séricas elevadas. Por eso es indispensable hacer un ultrasonido obstétrico en las primeras 12 semanas de gestación, para determinar si es un embarazo monocoriónico, como ocurre hasta en $30 \%$ de los embarazos gemelares, en los que hay mayor riesgo relativo de mortalidad, 2.5 veces mayor comorbilidad neurológica y riesgo de síndrome de transfusión gemelo-gemelo. ${ }^{9}$

De los embarazos gemelares, unidos o no, $75 \%$ llega a término antes de la semana 37 de gestación y cerca de $12 \%$ antes de la semana 32. Algunos estudios apoyan el uso de maduradores de la función pulmonar, aún sin contar con ningún indicio de actividad uterina, entre las semanas 32 a 34 . Hasta el momento no hay estudios que determinen la funcionalidad o no de dicha acción. ${ }^{1,10}$

El estudio de estos pacientes debe iniciarse en una etapa prenatal, que comienza con la búsqueda y la detección de malformaciones asociadas y de las estructuras anatómicas que se comparten, sobre todo cuando se trata de corazón único o fusión cerebral. En estos casos es necesario que los padres autoricen interrumpir el embarazo debido a las implicaciones de dichas alteraciones. ${ }^{1,9,10}$

Las teorías embriológicas más aceptadas indican que únicamente se pueden presentar entre la tercera y la cuarta semanas de la fertilización, debido a la fisión incompleta del óvulo fertilizado a causa de un defecto de la blastogénesis, lo cual determina el número y el grado de unión de las estructuras anatómicas que se comparten durante la formación. ${ }^{4}$ 
La etapa posnatal depende de las posibilidades de realizar una separación quirúrgica, sin tener un diagnóstico prenatal, lo que obligaría a tomar decisiones sobre tratamiento médico y quirúrgico de acuerdo con las condiciones clínicas al nacimiento, de tal modo que la única indicación de separación de urgencia es cuando uno de los gemelos está agonizando o muerto y se intenta mantener la vida del otro. Pocas situaciones médicas tienen problemas éticos tan relevantes como ésta, debido que el tratamiento implica la pérdida de órganos e incluso la vida de uno de los gemelos. Es importante recordar que todas las decisiones de tratamiento deben tomarse bajo los principios de autonomía, justicia, beneficencia, no maleficencia ${ }^{3}$ y que, además, de acuerdo con el artículo 4to. de la Constitución Política de los Estados Unidos Mexicanos, siempre se velará por el interés superior del menor.

Las decisiones éticas son parte muy importante del tratamiento de estos niños, pues son difíciles y requieren una consulta con varios especialistas y no sólo un juicio personal. Hay una constante tentación de realizar diversas técnicas posibles, que dañarían a uno de los gemelos. La regla primordial es que no todo lo que se puede hacer debe llevarse a cabo.

Las decisiones deben tener como base el bienestar de los menores, conocido ahora como el interés superior de los niños, sin discriminación; no ocasionar daño a ninguno y que los procedimientos tomen en cuenta por igual los derechos de ambos de acuerdo con los valores de nuestra cultura. Por ningún motivo uno de los niños con posibilidades, como era este caso, podría ser sacrificado para intentar salvar al otro. Aquí emergería la tentación eugenésica de vidas de menos valor, lo que para la medicina actual es inaceptable. La única posibilidad de que se intente salvar a uno a favor del otro, es que uno de ellos tuviese una enfermedad incurable y avanzada. El punto de vista de los padres con una información amplia, precisa y compasiva, es el más importante en la toma de decisiones médicas. Así ocurrió en este caso, en que las intervenciones médicas se orientaron al salvamento de los dos gemelos.

Un enfoque utilitarista de que uno de los menores debía ayudar al otro a sobrevivir, aun perdiendo la vida, no es aceptable porque los derechos de ambos son los mismos. El análisis de casos complejos como éste permite a los médicos una mejor toma de decisiones, más justa; nos recuerda que no somos omnipotentes y que la razón nos permitirá una actuación más prudente y compasiva.

La reciente introducción de la tomografía computada helicoidal multicorte combinada, utilizando estaciones de trabajo de procedimiento gráfico, nos permitió obtener imágenes tridimensionales volumétricas y multiplanares que determinan con gran precisión las anormalidades congénitas, complejas como en nuestro caso, que fueron valoradas cuidadosamente.

A pesar de que la inducción anestésica fue difícil, se decidió realizar una angiotomografía pues, a diferencia de la resonancia magnética, dicho estudio se realiza en un lapso de tiempo mucho más corto, con poca cantidad de medio de contraste y en forma simultánea en ambos gemelos. Las imágenes se valoraron en diferentes planos y se lograron obtener detalles anatómicos que fueron de gran utilidad para los médicos y los cirujanos durante la separación de los siameses.

Es importante aclarar que debido a las anomalías cardiacas del siamés 1 es difícil determinar si, en otras condiciones (no encontrándose unido a su gemelo), hubiese tenido mejor pronóstico. Este caso, en comparación con el caso descrito en el año 2005, en el cual las características físicas de los siameses permitieron su separación completa al encontrarse unidos por un puente de piel a 
nivel abdominal y un istmo en el hígado, sin contar con otras comorbilidades que pudieran poner en riesgo la vida de los gemelos, ${ }^{11}$ el uso de materiales novedosos como las mallas de titanio fue determinante para la supervivencia de 6 meses del siamés 2. Después de la separación el producto llegó al final de su vida por condiciones ajenas al abordaje inicial.

En México son pocos los casos publicados, el más significativo históricamente fue el de la separación quirúrgica de un par de siameses del sexo femenino en $1917^{12}$ en el que las gemelas sobrevivieron dos años a la separación.

\section{Agradecimiento}

Agradecemos al Dr. Armando Garduño Espinoza por los comentarios vertidos en este artículo.

\section{REFERENCIAS}

1. American College of Obstetricians and Gynecologists Committee on Practice Bulletins-Obstetrics, Society for Maternal-Fetal Medicine, ACOG Joint Editorial Committee ACOG Practice Bulletin \#56: Multiple gestation: complicated twin, triplet, and high-order multifetal pregnancy. Obstet Gynecol 2004;104(4):869-83.
2. Endres L, Wilkins I. Epidemiology and biology of multiple gestations. Clin Perinatol 2005;32(2):301-314.

3. Tovar JA. Gemelos unidos (siameses). Bol Pediatr 1998;38:259-263.

4. Usang E, Babatunde O, Archibong A, Udo J, Eduwen DA. Dicephalus parapagus conjoined twins discordant for anencephaly, a case report. J Med Case Rep 2010;5;4:38

5. Eagan J, Borgida A. Multiple gestations, the importance of ultrasound. Obstet Gynecol Clin North Am 2004;31(1):141158.

6. Corona Rivera JR, Corona Rivera E. Nosología actual de las duplicaciones embrionarias. Bol Med Hosp Infant Mex 2000;57(2):104-115.

7. Gilbert-Barness E, Raj P. Potter's pathology of the fetus infant and child. 2da ed. Geoffrey AM. Mosby an imprint of Elsevier, 2007, pp. 307-317.

8. Wigglesworth JS, Singer DB. Textbook of fetal and perinatal pathology. 2da ed. Baldwin VJ. Blackwell Scientific Publications, 1991, p. 251.

9. Modena $A B$, Bergella V. Antepartum management of multifetal pregnancies. Clin Perinatol. 2005;32(2):443454.

10. Elliot JP. Management of High order multiple Gestation. Clin Perinatol 2005;32(2):387-402.

11. Salgado JA, De la Torre L, López S, Ceballos C, Castillo A. Separación exitosa de gemelos unidos onfalópagos durante el periodo neonatal. Revista Mexicana de Cirugía Pediátrica, 2005;12(1):55-60.

12. Quijano Pitman F. La primera separación de hermanas siamesas Dr. Aureliano Urrutia 1917. Gac Med Mex 2000;136(2):170-71. 